\title{
Modélisation de la structure géométrique du maïs
}

\author{
L Prévot 1, F Aries 2, P Monestiez 2 \\ 1 INRA, station de bioclimatologie, 78850 Thiverval-Grignon; \\ 2 Station de biométrie, F-84140 Montfavet, France
}

(Reçu le 28 septembre 1990; accepté le 4 avril 1991)

\begin{abstract}
Résumé - Dans cette étude, on présente et teste un modèle permettant une description détaillée de la structure géométrique du maïs. Chaque élément de la plante est décrit à l'aide de relations paramétriques simples. Le pied est représenté par son nombre de feuilles et par les distances entre les points d'insertion de 2 feuilles successives. La feuille est décrite par un modèle de feuille développée et par un modèle de nervure centrale. La feuille développée est représentée par sa longueur, un paramètre reliant sa plus grande largeur à sa longueur et un paramètre de forme, lié à son degré d'engainement. La nervure centrale est modélisée par un arc de parabole et un arc d'ellipse, représentant ses parties ascendante et descendante. Ce modèle fait intervenir 6 paramètres de forme. Pour tester cette modélisation, on utilise des données acquises sur 56 plants de maïs. On a mesuré les dimensions de leurs feuilles, et les formes de leurs nervures centrales ont été numérisées. On présente et discute les distributions statistiques des paramètres obtenus par ajustement. Le modèle de feuille développée permet d'estimer la surface foliaire. Le modèle de nervure centrale montre une excellente aptitude à décrire la grande variété des formes observées. Ces 2 modèles permettent de calculer la fonction de distribution des angles foliaires avec une précision comparable à celle donnée par la méthode des silhouettes.
\end{abstract}

architecture de la végétation / modélisation / couvert végétal / Zea mays = maïs

Summary - A model of maize plant morphology. A model of maize plant morphology has been presented. The different plant parts are described with simple parametric equations: second order polynomials and conicals. The plant is represented by its number of leaves and by the vertical distance between 2 adjacent leaves which is related to its insertion height (fig 5; table l). The leaf is represented by a model of a fully-developed leaf (figs 2, 3) and by a shape model of the central vein (figs 1,4). The developed-leaf model involves leaf length which is related to its insertion height (fig 7; table III), a parameter relating its maximum width to its length (fig 6; table II) and a shape parameter, related to its sheathing (figs 3, 8, 9). The ascendant and descendant parts of the central vein are modeled as an arc of a parabola for the former and as an arc of an ellipse for the latter (fig 4). This model can be summarized by 6 shape parameters: the insertion angle of the leaf and parabola curvature, the curvilinear abscissa of the 2-arc junction and the angle formed by the 2 arcs, the elongation and the angular curvature of the ellipse. Discontinuity of the first derivative at the junction is allowed. In order to test the model, a data set of 56 maize plants is used. Leaf sizes were measured and the central vein shapes were digitalized (fig 1). The model is adjusted according to these experimental data, using a non-linear regression algorithm. The central vein model exhibits a very good ability to represent the various shapes observed (fig 10). Statistical distributions of the different model parameters are presented and discussed (fig 11). The developed-leaf model allows an estimation of leaf area (fig 12) and the whole model is used to estimate the leaf inclination distribution function of the canopy. Comparison with the results of the classical leaf profile method is satisfactory (fig 13).

canopy structure / modelling / plant canopy / Zea mays = maize

\section{INTRODUCTION}

Dans de nombreux domaines de la recherche agronomique, il est nécessaire de disposer d'une modélisation de la géométrie de la végétation : on peut citer, (entre autres) en ce qui concerne la partie aérienne des plantes, la quantification du rayonnement intercepté et, d'une façon plus générale, l'étude des relations avec le milieu environnant, l'approche des phénomènes de compétition, la description de la propagation des maladies.

Paradoxalement, la représentation de la géométrie des plantes est jusqu'à maintenant restée relativement fruste, se limitant à des indicateurs globaux : hauteur des plantes, surface foliaire, in- 
clinaison moyenne des feuilles, position et taille des organes reproducteurs.

Si l'on prend pour exemple l'étude des échanges radiatifs au sein de la végétation, 2 grands types de modélisation de l'architecture de la végétation coexistaient jusqu'à ces dernières années.

Les modèles géométriques (Brown et Pandolfo, 1969; Chiapale, 1975; Riou et al, 1989) assimilent la plante, ou un ensemble de plantes, à une forme géométrique simple - cylindre, sphère, parallélépipède, tronc de cône - généralement sans décrire les échanges à l'intérieur de cette forme; ils ne s'intéressent donc que peu à la structure géométrique fine de la végétation.

Les modèles statistiques représentent le couvert végétal comme un ensemble de strates (pour les modèles $1 \mathrm{D}^{*}$ : Suits, 1972; Kimes, 1984; Verhoef, 1984; Prévot, 1985), de tubes (pour les modèles 2D : Sinoquet, 1989; Sinoquet et Bonhomme, 1989) ou de parallélépipèdes (pour les modèles 3D : Kimes et Kirchner, 1982; Goel et Grier, 1988) à l'intérieur desquels les éléments du couvert ne sont connus, dans le meilleur des cas, que par les distributions statistiques de leurs caractéristiques (surface, orientation).

Plus récemment sont apparus des modèles (Aries et al, 1987; de Reffye et al, 1988) partant d'une description botanique de la plante - lois de croissance des entre-nœuds et lois de branchement, phyllotaxie, angles d'insertion - pour générer des maquettes informatiques tridimensionnelles de la végétation. Ces modèles, développés pour décrire des arbres ou arbustes, ne s'attachent généralement pas à une description détaillée des éléments du feuillage, ce qui limite leur utilisation pour des études telles que celle de l'interception du rayonnement par une culture herbacée.

Le travail présenté dans cet article se rattache à ces modèles botaniques, qui sont potentiellement les plus précis dans la description géométrique de la végétation.

Nous nous sommes attachés à développer un modèle capable de décrire de la manière la plus précise et la plus fidèle possible la géométrie d'une plante de grande culture, le maïs, choisi pour la relative simplicité des méthodes de détermination de sa structure et pour le grand nombre d'études expérimentales qui lui ont été consacrées. Nous nous sommes limités au cas de plantes ayant atteint leur plein développement végétatif, bien que les méthodes utilisées soient généralisables aux autres stades du développement de la plante. Cet article est principalement consacré à la description des feuilles : elles représentent en effet l'essentiel de la variabilité de la structure de la végétation et la modélisation de la forme des tiges et des épis ne pose pas de problème particulier.

La démarche utilisée consiste tout d'abord à établir des représentations paramétriques simples pour chacun des éléments de la plante: distances internodales, dimensions et forme de la feuille, forme dans l'espace de la nervure centrale de la feuille. Dans un deuxième temps, ces lois sont ajustées sur un ensemble de données expérimentales. Dans une dernière étape, on étudie les distributions statistiques des paramètres obtenus.

Par la suite, ces distributions pourront être utilisées pour générer des maquettes informatiques de plantes isolées, puis de peuplements, la démarche employée assurant la fidélité de la représentation.

\section{MATÉRIEL ET MÉTHODES}

\section{Dispositif expérimental et mesures}

Les données utilisées dans cet article ont été acquises au cours d'une expérimentation conduite en 1983 sur le site de l'INRA d'Avignon-Montfavet, dans le Sud-Est de la France $\left(4^{\circ} 51^{\circ} \mathrm{E}, 43^{\circ} 55^{\prime} \mathrm{N}\right)$.

Le maïs (Zea mays $L$ ) utilisé est de variété DEA, semé à 90000 pieds/ha et conduit selon des pratiques culturales classiques, une irrigation régulière permettant d'éviter les contraintes hydriques.

L'expérimentation s'est déroulée autour de la floraison femelle, survenue entre le 20 et le 25 juillet, de façon à obtenir une structure géométrique de la végétation suffisamment stable dans le temps, tout en conservant une variabilité de la forme des feuilles suffisante pour tester la souplesse des paramétrisations choisies.

La structure de la végétation est déterminée par la méthode des silhouettes (Bonhomme et VarletGrancher, 1978) : on prélève au hasard un certain nombre de plants dans la parcelle, chaque plant est photographié devant une mire quadrillée permettant la mise à l'échelle. Ces photographies sont ensuite numérisées, chaque feuille étant représentée par 8 à 30 points $(x i, y i)$ situés sur sa nervure centrale (voir fig 1 ).

\footnotetext{
* $1 \mathrm{D}:$ mono-dimensionnel, 2D : bi-dimensionnel, 3D : tri-dimensionnel.
} 


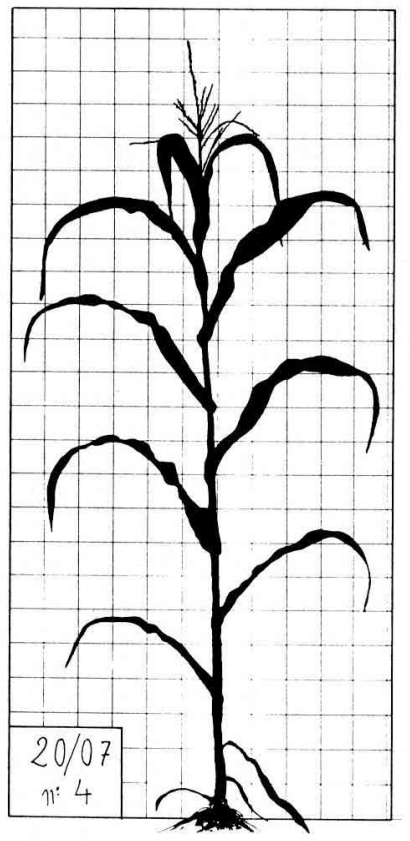

(a)

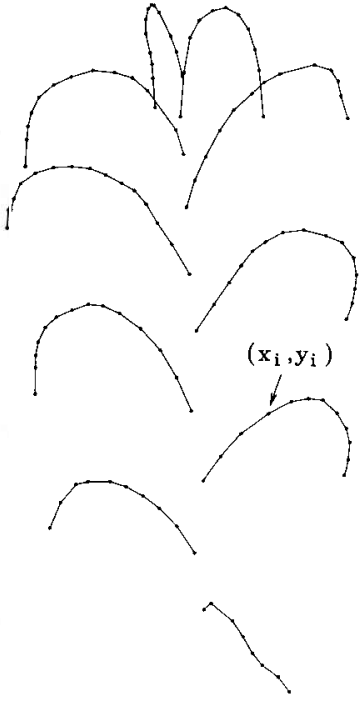

(b)
Fig 1. Méthode des silhouettes. Chaque plant est photographié devant une mire quadrillée (a). On numérise chaque feuille par une série de 8-30 points $\left(x_{i} ; y_{i}\right)$, situés le long de sa nervure centrale (b).

Rappelons que cette méthode est rendue possible car, dans le cas du maïs, les feuilles d'un pied sont approximativement situées dans un même plan vertical.

Deux séries de mesures ont été réalisées, le 20 juillet (30 plantes) et le 9 août (26 plantes), pour lesquelles on ne prenait en compte que les feuilles non sénescentes. Parallèlement, on a mesuré la longueur et la plus grande largeur de chaque feuille. La surface de l'ensemble de feuilles prélevées le 20 juillet a été déterminée par planimétrie optique.

Par ailleurs, on a mesuré tous les $10 \mathrm{~cm}$ la largeur de 35 feuilles provenant de 6 plants prélevés le 13 juillet. Pour ce faire, chaque feuille était posée à plat sur une mire quadrillée.

\section{Modèles et méthodes d'ajustement}

\section{Le pied}

Le pied est ici représenté par le nombre de feuilles et par les différences de hauteurs d'insertion entre 2 feuilles successives. La méthode des silhouettes permet de déterminer ces 2 caractéristiques. Signalons toutefois qu'il subsiste une certaine imprécision pour les feuilles encore partiellement engainées : on assimile dans ce cas le point d'insertion de la feuille à l'endroit où celle-ci se sépare de la tige.

Pour tenir compte des différences de taille pouvant exister entre les différents pieds, les hauteurs sont ici rapportées à la hauteur du point d'insertion de la der- nière feuille du pied. Dans la suite du texte, les hauteurs sont toujours adimensionnées de cette manière.

\section{Hauteur d'insertion des feuilles}

La distance entre les points d'insertion de 2 feuilles successives croît jusqu'à une hauteur proche de celle de l'épi, puis décroît ensuite. On représente son évolution avec la hauteur par un polynôme du second degré :

$$
\Delta y_{0 i}=\alpha_{1} y_{0 i}^{2}+\beta_{1} y_{0 i}+\gamma_{1}
$$

où $y_{0 i}$ est la hauteur du point d'insertion de la feuille de rang $i$ et $\Delta y_{0 i}$ est la distance $y_{0 i}-y_{0 i-1} . \alpha_{1}, \beta_{1}$ et $\gamma_{1}$ sont des coefficients ajustés par regression.

\section{La feuille développée}

La feuille développée, c'est-à-dire assimilée à une surface plane, est supposée symétrique par rapport à sa nervure centrale, assimilée à une droite. Pour les graminées, il est classique de relier la largeur $v$ de la feuille à la distance de son point d'insertion par une relation polynômiale : Sinoquet (1988) par exemple, représente la largeur de feuilles de maïs totalement dégainées par un polynôme du troisième degré. Nous avons choisi d'utiliser un polynôme du second degré car on s'intéresse ici à un ensemble de feuilles plus ou moins engainées et le gain en précision apporté par un polynôme de degré supérieur serait négligeable dans ce cas.

Ces relations sont en fait le plus souvent exprimées sous forme adimensionnelle : on effectue, pour chaque feuille, le changement de variables $u^{*}=u / u_{f}$ et $v^{*}=v / v_{f}$, où $u_{f}$ et $v_{f}$ sont respectivement la longueur et la plus grande largeur de la feuille (voir fig 2). On modélise alors la largeur $v^{*}$ à la distance $u^{*}$ de son point d'insertion sous la forme :

$$
v^{*}\left(u^{*}\right)=\alpha u^{\star 2}+\beta u^{\star}+\gamma
$$

À l'extrémité de la feuille, sa largeur étant nulle, on a : $\alpha+\beta+\gamma=0$

L'abscisse $u_{m}{ }^{*}$ correspondant à la plus grande largeur $v_{f}$ est soit le point correspondant à l'annulation de la dérivée de (2), soit l'origine $u_{m}{ }^{*}=0$;

- dans le premier cas, on a : $v_{m}{ }^{*}=\gamma-\beta^{2} /(4 \alpha)=1$, et les coefficients du polynôme (2) deviennent : $\beta=-2(\alpha$ $+\sqrt{-\alpha})$ et $\gamma=2 \sqrt{-\alpha+\alpha}$. La largeur à l'origine $v_{0}{ }^{*}=\gamma$

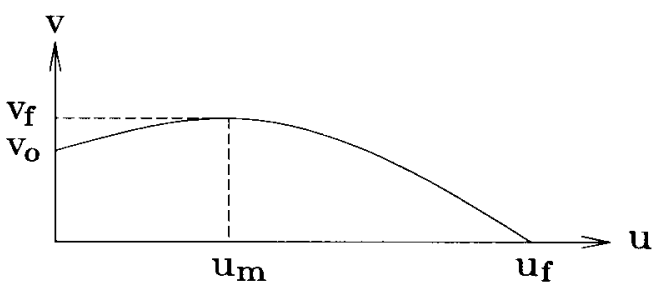

Fig 2. Forme de la feuille développée. $u_{f}$ et $v_{f}$ sont respectivement la longueur et la plus grande largeur de la feuille, $v_{0}$ est la largeur de la feuille à sa base, $u_{m}$ est l'abscisse correspondant à la plus grande largeur $v_{f}$ 
étant comprise entre 0 et $1, \alpha$ est compris entre -4 et -1 ;

- dans le deuxième cas, la largeur à l'origine $v^{*}{ }_{0}$ étant égale à 1 , les coefficients du polynôme (2) sont : $\beta=-(1+\alpha)$ et $\gamma=1$. Ce dernier cas n'existe que si la dérivée de (2) ne s'annule pas pour $u^{*}$ compris entre 0 et 1 , soit pour $\alpha$ compris entre -1 et 1 .

La prise en compte de ces différentes contraintes permet de réduire le nombre de paramètres de 3 à 1 . La forme de la feuille peut ainsi être représentée par le seul paramètre $\alpha$ et la fig 3 présente les différentes formes qu'on peut alors obtenir. Les faibles valeurs de $\alpha$ (inférieures à $-1,5$ ) correspondent à des feuilles dégainées, alors que des valeurs plus élevées correspondent à des feuilles encore partiellement engainées.

Le paramètre $\alpha$ est ajusté pour chaque feuille par minimisation de la somme des carrés des écarts entre modèle et mesures :

$$
\min \sum_{i=1 \ldots n}\left[\overline{v^{*}}\left(u_{i}^{*}\right)-v_{i}^{*}\right]^{2}
$$

où $v_{i}^{*}$ est la largeur adimensionnelle mesurée au point d'abscisse $\overline{v_{i}^{*}}$, et $u^{*}\left(v_{i}{ }^{*}\right)$ est donnée par le modèle (2).

\section{La nervure centrale}

\section{Modèle choisi}

Peu d'auteurs se sont consacrés à la modélisation de la forme de la nervure centrale de graminées. Baret (1986) représente la forme de feuilles de blé par 2 paraboles, l'une pour leur partie ascendante, l'autre pour leur partie descendante. Cette formulation ne permet toutefois pas de prendre en compte les rebroussements observés sur certaines feuilles de maïs fortement recourbées ou cassées.

Nous avons choisi de représenter la nervure centrale par des formes relativement simples, des arcs de coniques, de façon à minimiser le nombre de paramètres;

- la nervure est supposée être comprise dans un plan vertical, ce qui est assez bien respecté dans le cas du maïs;

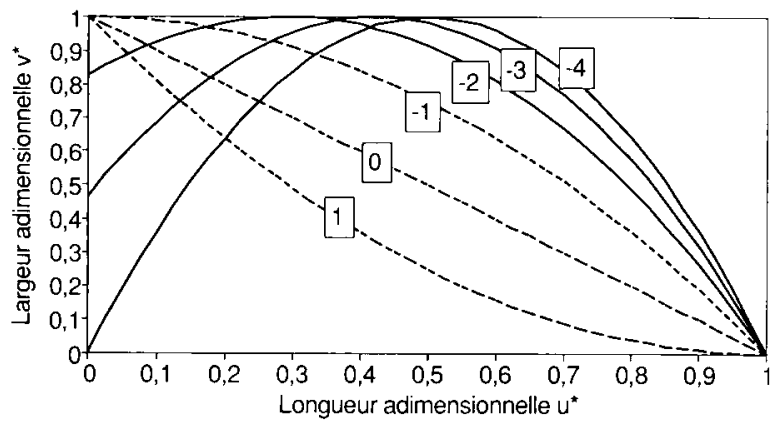

Fig 3. Modèle de forme de la feuille développée. Le modèle d'équation (2) permet de définir différentes formes de la feuille développée, selon la valeur du paramètre $\alpha$ (la représentation est faite en coordonnées adimensionnelles $u^{*}:=u$ / $u_{f}$ et $\left.v^{*}=v / v_{f}\right)$
- sa partie ascendante est assimilée à un arc de parabole à axe de symétrie vertical;

- sa partie descendante, lorsqu'elle existe, est assimilée à un arc d'ellipse dont l'un des axes est horizontal et l'autre vertical;

- le modèle est défini en plaçant l'origine du repère au point d'insertion de la feuille $\left(x_{0}, y_{0}\right)$ (fig 4$)$;

- l'équation de l'arc de parabole est :

$$
y=a x^{2}+b x
$$

avec $0 \leq x \leq x_{m}$ où $x_{m}$ est l'abscisse du point le plus haut de la feuille;

- l'équation de l'arc d'ellipse est, sous sa forme paramétrique :

$$
x=x_{c}+d \cos \varnothing ; y=y_{c}+e \sin \varnothing
$$

avec $\emptyset_{m} \leq \emptyset \leq \sigma_{f}$ où $\left(x_{c}, y_{c}\right)$ est le centre de l'ellipse, $d$ et $e$ sont les longueurs de ses demi-axes horizontal et vertical, $\sigma_{m}$ et $\sigma_{f}$ sont les angles paramétriques correspondant aux 2 extrémités de l'arc.

- le raccordement les 2 modèles est situé au point le plus haut de la feuille $\left(x_{m}, y_{m}\right)$, où l'on impose la contrainte de continuité :

$$
x_{m}=x_{c}+d \cos \sigma_{m} ; a x_{m}^{2}+b x_{m}=y_{c}+e \sin \sigma_{m}
$$

Par contre, on n'impose pas de contrainte sur la dérivée au point de raccordement $\left(x_{m}, y_{m}\right)$, un certain nombre de feuilles étant cassées à cet endroit;

- le modèle comporte ainsi 9 paramètres qui, outre la position du point d'insertion $\left(x_{0}, y_{0}\right)$, se décomposent en :

- 3 paramètres pour la partie ascendante de la feuille : $a, b$, et $x_{m}$ par exemple;

- 6 paramètres pour la partie descendante de la feuille : $x_{c}, y_{c}, d, e, \sigma_{m}$ et $\sigma_{f}$ par exemple;

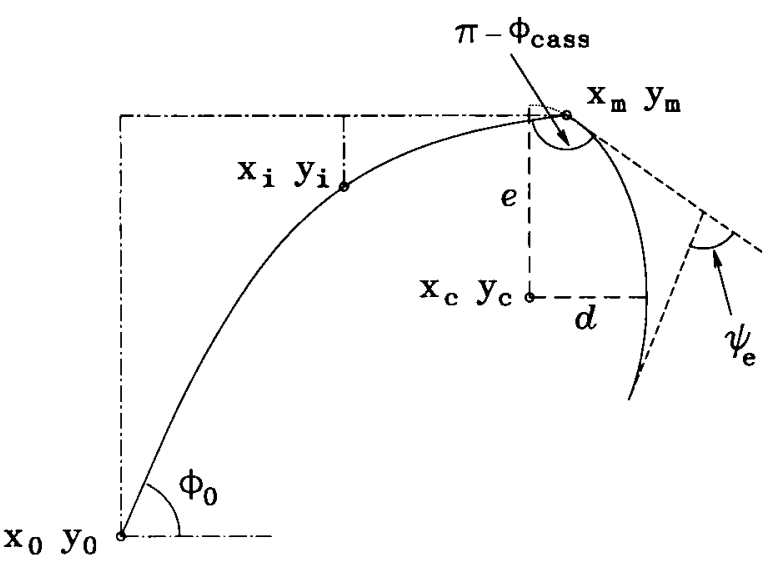

Fig 4. Modèle de forme de la nervure centrale. La partie ascendante de la feuille, comprise entre le point d'insertion sur la tige $\left(x_{0}, y_{0}\right)$ et le point d'ordonnée maximale $\left(x_{m}, y_{m}\right)$ est représentée par un arc de parabole (éqn 3). Sa partie descendante, comprise entre le point $\left(x_{m}, y_{m}\right)$ et son extrémité distale est représentée par un arc d'ellipse de centre $\left(x_{c}, y_{d}\right)$ (éqn 4). 
- la condition de continuité réduit le nombre total de paramètres de 9 à 7 .

\section{Ajustement du modèle}

Pour chacun des 2 sous-modèles, arc de parabole et arc d'ellipse, le critère de minimisation utilisé pour l'ajustement est la distance entre les arcs de chaque conique et le nuage des $n$ points expérimentaux ( $x i$, $y i), i=1 \ldots n$, obtenus par la méthode des silhouettes. La distance verticale (selon l'axe des $y$ ), classiquement utilisée comme critère de minimisation, ne peut être employée ici car elle n'est pas adaptée au cas des paraboles proches de la verticale et est inutilisable dans le cas de l'ellipse, qui n'est pas définie comme une fonction $y=f(x)$.

Soit $P_{\Theta}$ l'arc de parabole et $\varepsilon_{\Theta}$ l'arc d'ellipse, $\Theta$ étant le vecteur des paramètres $\left(\Theta_{1}, \Theta_{2} \ldots \Theta_{7}\right)$, critère de minimisation utilisé est :

$$
\min \left[\sum_{i=1}^{i_{\max }} D^{2}\left(P_{\Theta},\left(x_{i}, y_{i}\right)\right)+\sum_{i=i_{\max }+1}^{n} D^{2}\left(\varepsilon_{\Theta},\left(x_{i}, y_{i}\right)\right)\right]
$$

où $D^{2}\left(A_{\Theta},\left(x_{i}, y_{i}\right)\right)$ est la distance entre l'arc de conique $A_{\Theta}$ et le point observé $\left(x_{i}, y_{i}\right), i_{\max }$ est le rang du point mesuré d'ordonnée maximale $\left(x_{m}, y_{m}\right), n$ est le nombre de points observés.

Si l'arc $A_{\Theta}$ est continu et peut s'écrire sous la forme paramétrique $x=f_{A}(t), y=g_{A}(t)$, avec $t_{\min } \leq t \leq t_{\max }$, le point $P(t)$ de l'arc le plus proche du point observé $\left(x_{i}\right.$, $\left.y_{i}\right)$ est soit le point correspondant à $t_{\min }$, soit le point correspondant à $t_{\max }$, soit le point correspondant à $t$ solution de:

$$
\frac{\partial\left(\left(f_{A}(t)-x_{i}\right)^{2}+\left(g_{A}(t)-y_{i}\right)^{2}\right)}{\partial t}=0
$$

Dans la pratique, la résolution de (6) se ramène à trouver les racines d'un polynôme du troisième degré pour l'arc de parabole, du quatrième degré pour l'arc d'ellipse. On résout l'équation (6) dans l'intervalle [ $t_{\text {min }}$, $t_{\text {max }}$ ] et l'on vérifie que les solutions correspondant aux bornes $t_{\min }$ et $t_{\max }$ de l'arc ne sont pas plus proches.

La minimisation du critère (5) est un problème non linéaire que l'on résout par la méthode de Marquardt (1963).

\section{Reparamétrisation du modèle}

La première paramétrisation du modèle de nervure centrale est peu adaptée à l'étude des distributions de ses paramètres. En effet, certains d'entre eux $\left(a, b, x_{G}\right.$, $y_{c}$, $d$ et $e$ ) peuvent tendre vers l'infini en valeur absolue pour des arcs de parabole quasi-verticaux ou des arcs d'ellipse quasi-rectilignes. Des valeurs très grandes, positives ou négatives, pourront ainsi indifféremment être choisies pour approcher des courbes semblables et il est impossible d'estimer ne serait-ce qu'une espérance pour chaque paramètre. Nous avons donc repa- ramétré le modèle en utilisant des grandeurs dérivées de sa première définition, mais choisies de telle sorte qu'elles soient finies et correspondent à des paramètres plus facilement interprétables (fig 4). Ces paramètres sont :

\section{La longueur de la feuille $v_{f}$}

\section{L'angle d'insertion de la feuille $\Phi_{0}$}

Il est défini comme l'angle d'inclinaison de la feuille à son point d'insertion.

\section{La courbure de la parabole $C_{p}$}

Elle est définie par le paramètre $c_{p}=y_{m} / y_{i}$, où $y_{m}$ est la hauteur du point le plus haut de la feuille (raccordement des 2 modèles) et $y_{i}=a x_{i}^{2}+b x_{i}$ avec $x_{i}=x_{m} / 2$. La valeur $C_{p}=2$ correspond par définition à une droite. L'arc de parabole étant strictement ascendant, la courbure $C_{p}$ a pour limite inférieure la valeur $4 / 3$, correspondant à une feuille horizontale au niveau du point de raccordement, et pour limite supérieure la valeur 4 , correspondant à une feuille horizontale au niveau du point d'insertion.

\section{La position du point de raccordement des 2 modèles $P_{\text {cass }}$}

On caractérise la position sur la nervure du point de raccordement $\left(x_{m}, y_{m}\right)$ par le paramètre $P_{c a s s}$, égal au rapport de la longueur de l'arc de parabole à la longueur totale $v_{f}$ de la feuille. II s'agit donc d'une abscisse curviligne adimensionnelle : une feuille entièrement ascendante correspond à $P_{\text {cass }}=1$, une feuille entièrement descendante correspond à $P_{\text {cass }}=0$.

\section{L'angle de cassure de la feuille $\Phi_{\text {cass }}$}

Le point de raccordement $\left(x_{m}, y_{m}\right)$ peut correspondre à une cassure de la feuille, puisque l'on n'impose pas de condition de continuité de la dérivée du modèle en ce point. L'angle de cassure $\Phi_{\text {cass }}$ est défini comme le complément à $\pi$ de la différence entre les pentes de la parabole et de l'ellipse, au point de raccordement : les feuilles non cassées sont caractérisées par $\Phi_{\text {cass }}$ $=0^{\circ}$.

\section{L'allongement de l'ellipse $\varepsilon$}

On le caractérise par le paramètre $\varepsilon$ défini par :

$$
\varepsilon=\mid \begin{array}{ll}
+\left(o^{2}-e^{2}\right) / \alpha^{2} & \text { pour } d \geq e \\
-\left(e^{2}-o^{2}\right) / e^{2} & \text { pour } d<e
\end{array}
$$

où $d$ et $e$ sont respectivement les longueurs des demi-axes horizontal et vertical de l'ellipse. II s'agit donc du carré de l'excentricité de l'ellipse, affecté d'un signe différenciant les ellipses à allongement vertical $(\varepsilon<0)$ des ellipses à allongement horizontal $(\varepsilon>0)$. 


\section{La courbure angulaire de l'ellipse $\psi_{e}$}

Elle est définie comme la différence angulaire entre les pentes des 2 extrémités de l'ellipse. Les ellipses quasiment rectilignes sont caractérisées par de faibles valeurs de $\psi_{e}$ et les ellipses très recourbées par de fortes valeurs de $\psi_{\mathrm{e}}$. Cette définition est indépendante de la longueur de l'arc d'ellipse.

Cette paramétrisation est équivalente à celle définie par les 7 paramètres des équations (3) et (4) mais ne pose pas les mêmes problèmes puisque les nouveaux paramètres sont par définition des grandeurs finies.

\section{RÉSULTATS ET DISCUSSION}

\section{Analyse des paramètres obtenus}

\section{Le pied}

\section{Nombre de feuilles par pied}

Rappelons que seules les feuilles visibles et non sénescentes sont ici prises en compte. Le nombre de feuilles par plant varie entre 8 et 12 pour le 20 juillet (moyenne : 9,9 ) et entre 7 et 10 pour le 9 août (moyenne : 8,7). Entre les 2 dates, un certain nombre de feuilles ont en effet été cassées à leur base par le vent. Celles-ci ne sont donc plus prises en compte.

\section{Hauteur d'insertion des feuilles}

La figure 5 présente l'évolution de la distance entre les points d'insertion de 2 feuilles successives, ainsi que le modèle d'équation (1) ajusté sur ces données. Les coefficients obtenus par régression sont donnés au tableau $\mathrm{l}$.

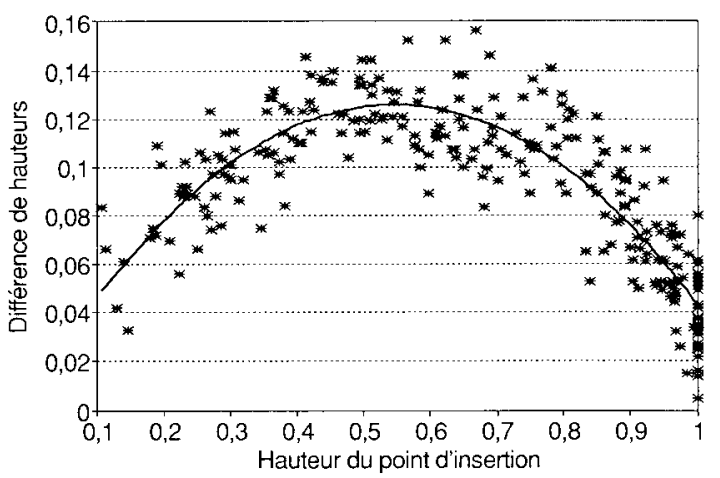

Fig 5. Évolution de la distance entre les points d'insertion de deux feuilles successives. On représente la différence de hauteurs entre les points d'insertion de 2 feuilles successives d'un même plant, en fonction de la hauteur du point d'insertion, adimensionnée par la hauteur du point d'insertion de la dernière feuille du plant. " : points expérimentaux; - : modèle d'équation (1).
Tableau I. Coefficients de l'équation (1) obtenus par régression.

\begin{tabular}{lrl}
\hline Coeff & Moyenne & Écart type \\
\hline$\alpha_{1}$ & $-0,40$ & 0,02 \\
$\beta_{1}$ & 0,44 & 0,02 \\
$\gamma_{1}$ & 0,07 & 0,02 \\
$R^{2}$ & 0,755 & $d d l=260$ \\
\hline
\end{tabular}

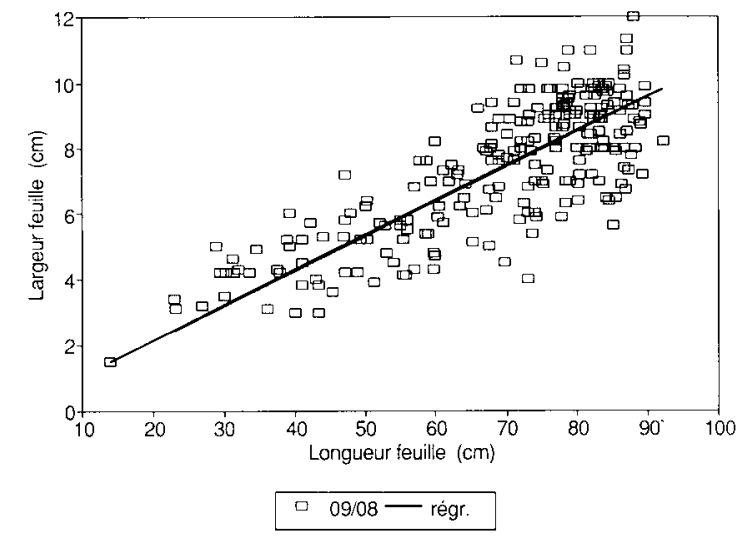

Fig 6. Relation entre la longueur et la largeur des feuilles. On représente la relation existant entre la longueur $u_{f}$ et la plus grande largeur $v_{f}$ des feuilles sous la forme linéaire d'équation (7). $\square$ : feuilles du 0,9 août; - : droite de régression.

\section{La feuille développée}

\section{Dimensions de la feuille}

La figure 6 présente la relation existant entre la largeur maximale $v_{f}$ d'une feuille et sa longueur $u_{f}$, pour l'ensemble des feuilles étudiées. On représente cette relation sous la forme linéaire :

$$
v_{f}=\alpha_{2} u_{f}+\beta_{2}
$$

La méthode des silhouettes conduit à estimer la largeur des feuilles connaissant leur longueur, aussi on utilise une régression par les moindres carrés pour estimer les coefficients de cette relation. Elle est ajustée sur l'ensemble des feuilles du 9 août et les coefficients sont donnés dans le tableau II. L'ordonnée à l'origine $\beta_{2}$ n'étant pas significativement différente de 0 , on calcule le coefficient $\alpha_{2}$ en imposant une origine nulle (voir tableau II).

La figure 7 donne l'évolution de la longueur $u_{f}$ de la feuille en fonction de la hauteur de son point d'insertion $y_{0}$. La longueur croît jusqu'à une 
Tableau II. Coefficients de l'équation (7) obtenus par régression.

\begin{tabular}{|c|c|c|c|c|}
\hline \multirow[b]{2}{*}{ Coeff } & \multicolumn{2}{|c|}{ Origine $\beta_{2}$ calculée } & \multicolumn{2}{|c|}{ Origine $\beta_{2}=0$} \\
\hline & Moyenne & Ecart type & Moyenne & Écart type \\
\hline$\alpha_{2}$ & 0,098 & 0,005 & 0,106 & 0,001 \\
\hline$\beta_{2}$ & 0,62 & 1,26 & - & - \\
\hline$R^{2}$ & 0,630 & $d d l=234$ & 0,625 & $d d l=233$ \\
\hline
\end{tabular}

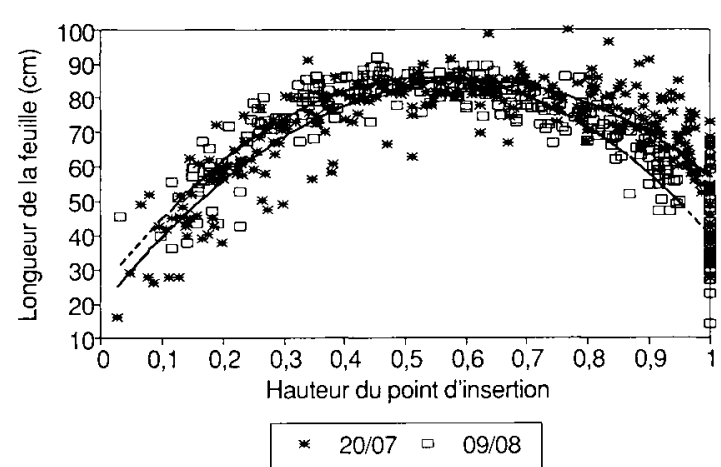

Fig 7. Évolution de la longueur des feuilles avec la hauteur de leur point d'insertion. La hauteur du point d'insertion est adimensionnée par la hauteur du point d'insertion de la dernière feuille du plant. * : feuilles du 20 juillet; $\square$ : feuilles du 09 août; --- : modèle d'éq (8) pour le 20 juillet; - : modèle d'éq (8) pour le 09 août.

hauteur proche de celle de l'épi, puis décroît ensuite. On représente cette évolution sous la forme parabolique :

$$
u_{f}=\alpha_{3} y_{0}^{2}+\beta_{3} y_{0}+\gamma_{3}
$$

Les coefficients obtenus par régression sont donnés dans le tableau III.

Tableau III. Coefficients de l'équation (8) obtenus par régression.

\begin{tabular}{lrrrrr}
\hline & \multicolumn{2}{c}{20 juillet } & & \multicolumn{2}{c}{ O9 août } \\
\cline { 6 - 6 } \cline { 5 - 6 } Coeff & Moyenne & Écart type & & Moyenne Écart type \\
& & & & & \\
\hline$\alpha_{3}$ & $-180,5$ & 7,0 & & $-214,9$ & 6,5 \\
$\beta_{3}$ & 218,0 & 8,3 & & 230,8 & 7,8 \\
$\gamma_{3}$ & 19,5 & 8,5 & & 24,1 & 6,5 \\
$R^{2}$ & 0,698 & $d d l=297$ & & 0,850 & $d d l=232$ \\
\hline
\end{tabular}

\section{Forme de la feuille}

Le modèle de forme développée de la feuille donné par l'équation (2) a été ajusté sur chacune des 35 feuilles prélevées le 13 juillet et on trouvera en figure 8,3 exemples de comparaison des largeurs de feuilles estimées par le modèle et mesurées. L'écart type résiduel des largeurs est de $0,4 \mathrm{~cm}$, pour 278 observations.

La figure 9 donne la répartition des paramètres $\alpha$ ainsi obtenus. Le paramètre $\alpha$ varie entre $-2,8$ pour les feuilles les plus dégainées et $-0,9$ pour les plus engainées.

(a)

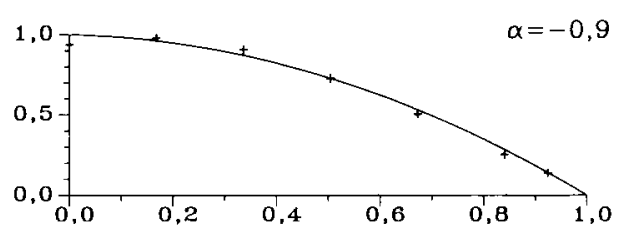

(b)

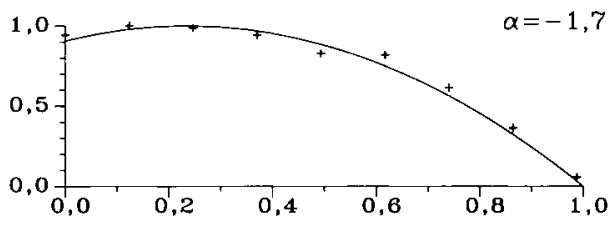

(c)

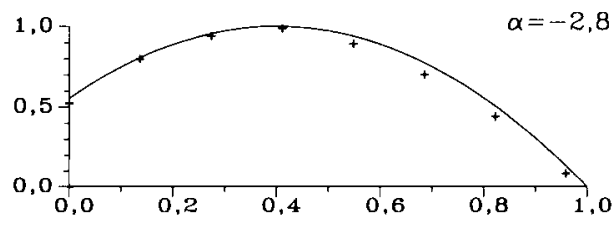

Fig 8. Ajustement du paramètre de forme $\alpha$. Exemples d'ajustement du modèle de largeur de feuille (2). En abscisse : distance adimensionnelle au point d'insertion $u^{*}$, en ordonnée : largeur adimensionnelle $v^{*}$. (a) $\alpha=-0,9$ : feuille engainée; (b) $\alpha=-1,7$ : feuille partiellement dégainée; (c) $\alpha$ $=-2,8$ : feuille totalement dégainée; + : largeurs observées; - : modèle d'équation (2).

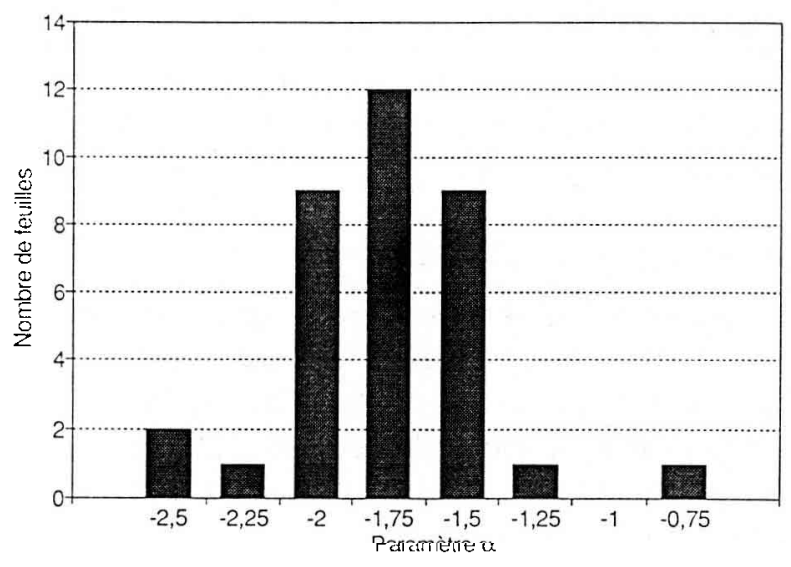

Fig 9. Distribution du paramètre de forme $\alpha$. Nombre de feuilles ayant une forme développée définie par une valeur donnée du paramètre $\alpha$ (ajustement du modèle d'équation (2) sur 35 feuilles prélevées le 13 juillet). 


\section{La nervure centrale}

\section{Ajustement du modèle}

Le modèle de forme de la nervure centrale défini par les équations (3) et (4) a été ajusté sur chacune des 533 feuilles disponibles (20 juillet et 09 août). La comparaison entre les courbes ajustées et les données de départ permet de s'assurer de la très bonne adéquation du modèle dans la plupart des cas. Seule une vingtaine de feuilles s'écarte sensiblement du modèle : il s'agit soit de feuilles rabattues ou cassées par le vent, soit de feuilles ayant une partie verticale de type hyperbolique, pour lesquelles l'approximation par un segment de parabole n'est pas tout à fait adéquate, sans toutefois beaucoup s'éloigner des points expérimentaux.

La figure 10 donne quelques exemples de plants de maïs sur lesquels le modèle a été ajusté. On notera sa capacité à décrire des formes de nervures très variées, malgré le nombre relativement limité de ses paramètres. L'écart type résiduel calculé pour chaque feuille varie entre 0,0 et $3,5 \mathrm{~cm}$, sa valeur moyenne est de $0,5 \mathrm{~cm}$.

La comparaison des longueurs de feuilles calculées par le modèle et déduites des silhouettes numérisées s'avère elle aussi excellente : la moyenne des résidus longueur observée - longueur estimée est de $8 \mathrm{~mm}$ (écart type de $8 \mathrm{~mm}$ ) pour une longueur moyenne de $66 \mathrm{~cm}$.

\section{Analyse des paramètres obtenus par ajustement}

Deux de ces paramètres, la longueur de la feuille $v_{f}$ et la hauteur de son point d'insertion $y_{0}$, ont déjà été discutés précédemment.

\section{Abscisse du point d'insertion $x_{0}$}

Dans la partie inférieure de la végétation, les variations de $x_{0}$ sont de l'ordre de l'épaisseur des tiges (quelques $\mathrm{cm}$ ). Au sommet du couvert, la variabilité de $x_{0}$ augmente légèrement, avec la courbure de certaines tiges.

\section{Angle d'insertion des feuilles $\Phi_{\circ}$}

La figure 11 (a) donne la répartition de l'angle d'inclinaison des feuilles à leur point d'insertion (angle d'inclinaison par rapport à l'horizontale). Une grande majorité de feuilles $(80 \%)$ ont un angle d'insertion compris entre 45 et $75^{\circ}$. À la date du 20 juillet, on note une légère corrélation positive entre l'angle d'insertion $\Phi_{0}$ et la hauteur du point d'insertion $y_{0}$. Cette relation disparaît le 09 août.

\section{Courbure de la parabole}

Sur la figure 11 , on constate que près des deux tiers des feuilles sont horizontales au niveau de leur point le plus élevé (courbure $C_{p}$ proche de $4 / 3)$. Environ un tiers des feuilles, surtout situées dans le haut du couvert, s'en éloignent tout en gardant un concavité dirigée vers le bas $(4 / 3<$ $\left.C_{p}<2\right)$. Seule une minorité de feuilles $(7,5 \%)$ présentent une concavité dirigée vers le haut (2 $\left.<C_{p}<4\right)$.

\section{Raccordement des modèles}

On constate sur la figure $11 \mathrm{c}$ donnant la répartition du paramètre $P_{\text {cass }}$ qu'il est compris entre 0,45 et 0,70 pour plus de la moitié des feuilles.
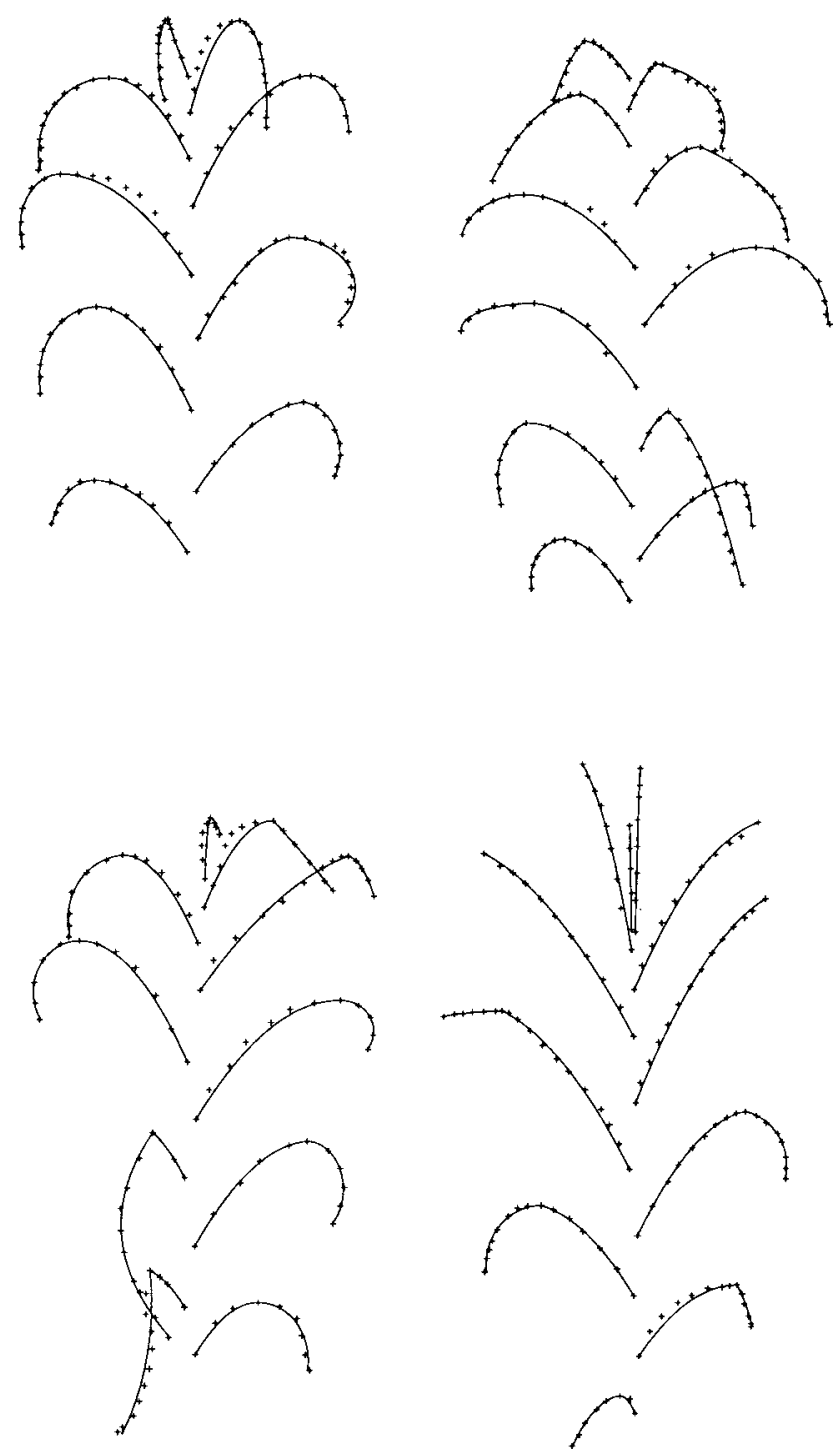

Fig 10. Ajustement du modèle de nervure centrale. Quatre exemples de plants sur les feuilles desquels le modèle de nervure centrale a été ajusté. + : points $\left(x_{i}, y_{i}\right)$ observés; - : modèle de nervure centrale. 


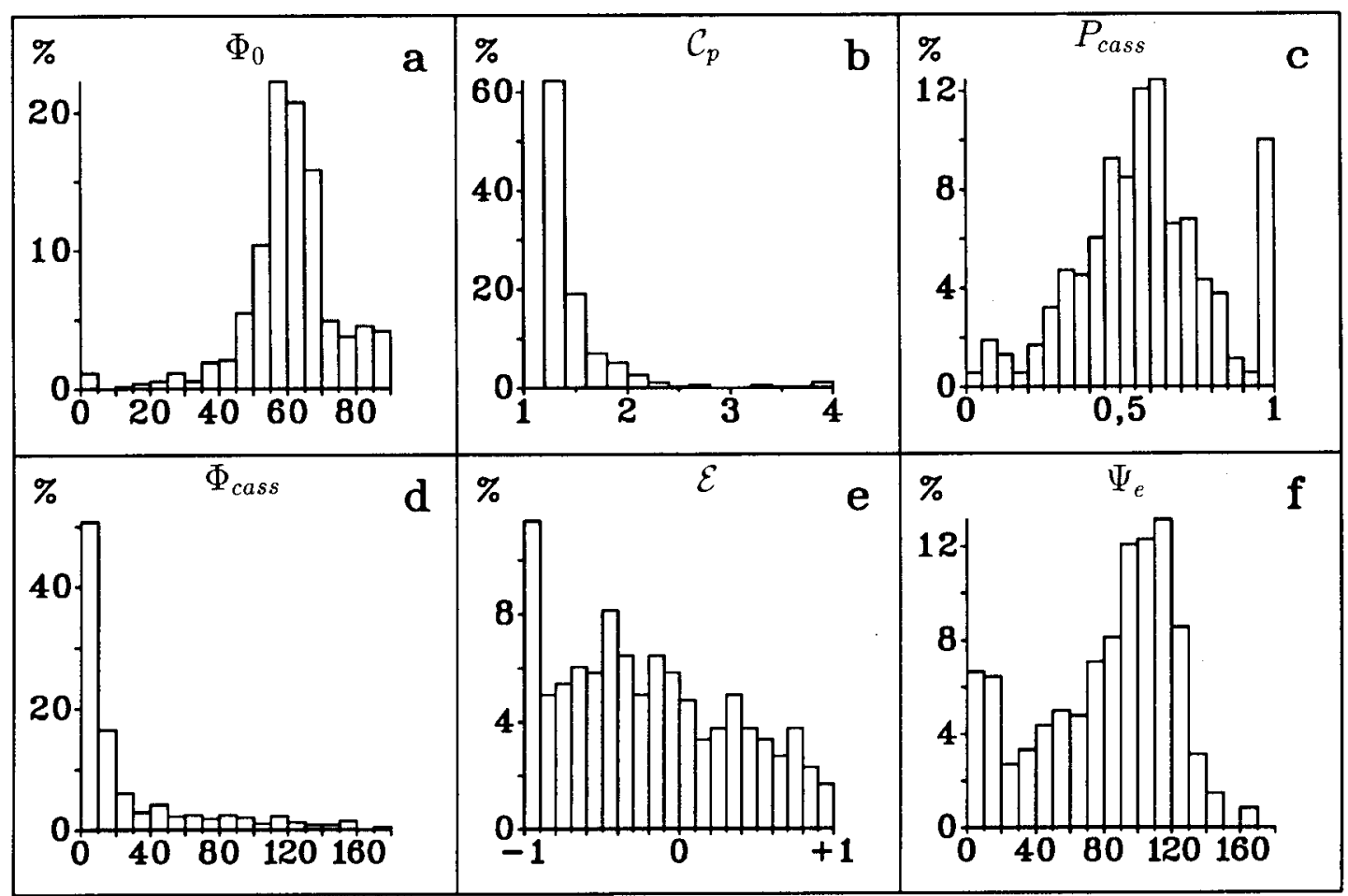

Fig 11. Distributions des paramètres de forme de la nervure centrale, exprimées en pourcentage du nombre total de feuilles (a, $\mathbf{b}$ et c) ou du nombre de feuilles représentées par un arc de parabole et un arc d'ellipse (d, e et f). (a) : distribution de l'angle d'insertion $\Phi_{0} ;$ (b) : distribution du paramètre de courbure des arcs de parabole $C_{p} ;(c)$ : distribution du point de changement de modèle $P_{\text {cass }}$; (d) : distribution de l'angle de cassure $\Phi_{c a s s} ;($ ) distribution de l'allongement des arcs d'ellipse $\varepsilon$; (f) : distribution de la courbure angulaire des arcs d'ellipse $\psi_{\mathrm{e}}$.

Le point de raccordement se rapproche du point d'insertion entre le 20 juillet $\left(0,49<P_{\text {cass }}<0,75\right.$ pour $50 \%$ des feuilles $)$ et le 09 août $\left(0,40<P_{\text {cass }}\right.$ $<0,65$ pour $50 \%$ des feuilles).

\section{La proportion de feuilles exclusivement ascendantes}

Ces feuilles représentées par $P_{\text {cass }}=1$ sont en proportion décroissante entre le 20 juillet où elle vaut 0,13 et le 09 août où elle vaut 0,06 (la différence est significative au seuil de $1 \%$ ). À la date du 20 juillet, ces feuilles sont surtout situées dans la partie haute de la végétation : les moyennes des hauteurs adimensionnelles des points d'insertion $y_{0}$ de ces feuilles est de 0,80 contre 0,58 pour les autres feuilles (la différence est significative au seuil de $1 \%$ ). À la date du 09 août, cette différence n'est pas significative : 0,68 contre 0,61 , mais le nombre de feuilles exclusivement ascendantes est très réduit (14).

\section{Angle de cassure $\Phi_{\text {cass }}$}

La figure $11 \mathrm{~d}$ présente la répartition de l'angle de cassure $\Phi_{\text {cass. }}$. Plus de la moitié des feuilles ne sont pas cassées $\left(\Phi_{\text {cass }}<10^{\circ}\right)$ et un quart des feuilles ne sont que faiblement cassées $\left(10^{\circ}\right.$ $<\Phi_{\text {cass }}<35^{\circ}$ ).

\section{Allongement de l'ellipse}

La figure 11 e présente la répartition du paramètre d'allongement $\varepsilon$. Les deux tiers des ellipses ont un allongement marqué $(|\varepsilon|>0,4)$ et les ellipses à allongement vertical $(\varepsilon<0)$ sont les plus nombreuses $(60 \%)$. Ces 2 caractéristiques s'accentuent du 20 juillet au 9 août.

\section{Courbure angulaire de l'ellipse $\psi_{e}$}

Sur la figure $11 \mathrm{f}$ qui donne la répartition des courbures $\psi_{e}$, se distinguent 2 familles de feuilles : des feuilles à faible courbure $\left(\psi_{e}<30^{\circ}\right)$ et des feuilles à courbure relativement forte $\left(\psi_{e}\right.$ $\left.>30^{\circ}\right)$. Une analyse plus détaillée montre que si les 2 types de feuilles se rencontrent dans l'ensemble du couvert végétal, on trouve plus d'ellipses quasi rectilignes $\left(\psi_{e}<25^{\circ}\right)$ au sommet de la végétation, correspondant à des feuilles jeunes. Les feuilles pleinement développées ont des courbures comprises entre 80 et $130^{\circ}$. 


\section{Application à l'estimation de la fonction de distribution des angles foliaires}

La fonction de distribution des angles foliaires répartition de la surface foliaire par classes d'inclinaison des feuilles - est un paramètre important pour décrire les échanges radiatifs au sein des couverts végétaux. Elle peut être calculée à partir de la méthode des silhouettes, qui a été conçue dans ce but (Bonhomme et VarletGrancher, 1978) : il faut pour ce faire d'une part, disposer d'une estimation de la surface foliaire et d'autre part, connaître la répartition des angles des feuilles.

\section{Estimation de la surface foliaire}

II est classique d'utiliser des relations statistiques entre la surface d'une feuille et ses dimensions linéaires pour estimer l'indice foliaire d'un couvert végétal (voir par ex Daughtry et Hollinger, 1984). Ces relations supposent, implicitement ou explicitement, l'existence d'un modèle de forme de la feuille tel que celui décrit par l'équation (2).

Connaissant les dimensions $u_{f}$ et $v_{f}$ de chaque feuille, ainsi que leur forme donnée par le paramètre $\alpha$, on peut calculer leur surface $S$ par :

$$
S=S^{*}(\alpha) u_{f} v_{f}
$$

où $S^{*}(\alpha)$ est la suface adimensionnelle définie par l'intégration du modèle de largeur (2) :

$$
\begin{aligned}
& S^{*}(\alpha)=\int_{u^{*}=0}^{1} v^{*} d u^{*}=\alpha / 3+\beta / 2+\gamma \\
& S^{*}(\alpha)=\alpha / 3+\sqrt{ }-\alpha \text { pour }-4 \leq \alpha \leq-1 \\
& S^{*}(\alpha)=(1-\alpha / 3) / 2 \text { pour }-1 \leq \alpha \leq-1
\end{aligned}
$$

On notera que la surface adimensionnelle $S^{*}(\alpha)$ est relativement peu sensible à la valeur du paramètre de forme $\alpha$. Pour les valeurs courantes de celui-ci, elle varie entre 0,75 (pour $\alpha=-2,25$ ) et 0,72 (pour $\alpha=-1,50$ ). De plus, elle est indépendante des dimensions $u_{f}$ et $v_{f}$ des feuilles.

On définit la surface adimensionnelle moyenne $\overline{S^{*}}$ comme la moyenne des surfaces adimensionnelles $S^{*}$, pondérée par la surface des feuilles, c'est-à-dire par le produit $u_{f} v_{f}$. Elle est dans notre cas égale à 0,749 . À partir des relations (7) et (9), on peut donc estimer la surface $S$ des feuilles, connaissant leur seule longueur $u$, par :

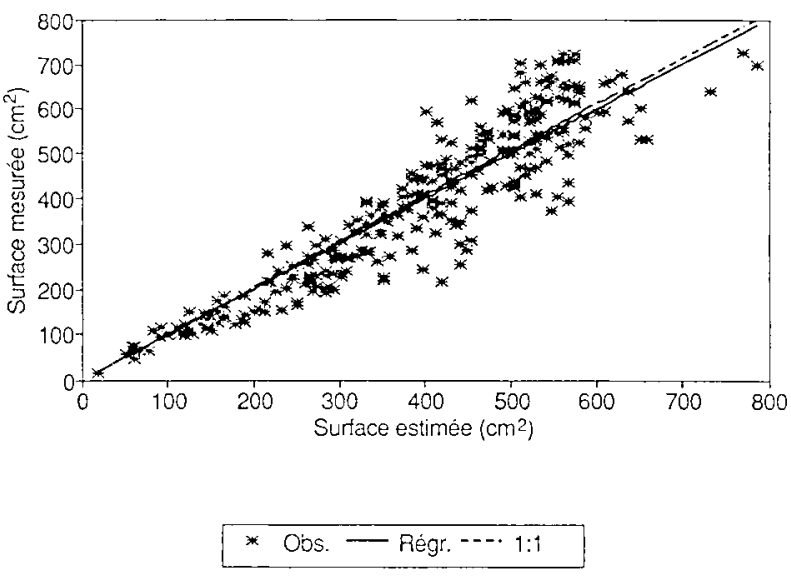

Fig 12. Estimation de la surface des feuilles : validation. L'équation (10), dérivée des équations (7) et (9), respectivement ajustées sur les feuilles du 09 août et du 13 juillet, est appliquée aux feuilles du 20 juillet. ${ }^{*}$ : feuilles du 20 juillet; droite de régression; --- : droite de pente :1:1.

$$
S=\overline{S^{\star}} u_{f} v_{f}=\overline{S^{\star}} \alpha_{2} u_{f}^{2}
$$

La figure 12 présente la validation de cette estimation. La pente de la droite de régression entre surfaces estimées et surfaces mesurées n'est pas significativement différente de 1 et son ordonnée à l'origine n'est pas significativement différente de 0 (au seuil de $5 \%$ ). Les coefficients $\overline{S^{*}}$ et $\alpha_{2}$ ayant été ajustés sur des données indépendantes de celles utilisées pour cette validation, le modèle de surface (10) est donc validé.

La surface d'une feuille est donc calculée à partir de sa longueur, qui peut être déterminée par la méthode des silhouettes ou estimée à partir de la hauteur de son point d'insertion.

\section{Distribution des angles foliaires}

Nous avons testé le modèle de nervure centrale en comparant les fonctions de distributions des angles foliaires déduites des mesures brutes des silhouettes (méthode classique) et déduites du modèle. Pour réaliser ce calcul, il faut disposer d'une relation entre la largeur de la feuille et la distance à son point d'insertion telle que celle donnée par l'équation (2). Pour chaque feuille, la nervure centrale modélisée a été discrétisée (50 points pour la partie parabolique et 50 points pour la partie ellipsoïdale). Le calcul a de plus été effectué pour 3 couches horizontales de végétation ayant chacune une épaisseur de $0,8 \mathrm{~m}$.

On trouvera en figure 13 les résultats de cette estimation de la fonction de distribution des angles foliaires. La végétation a un port érigé 

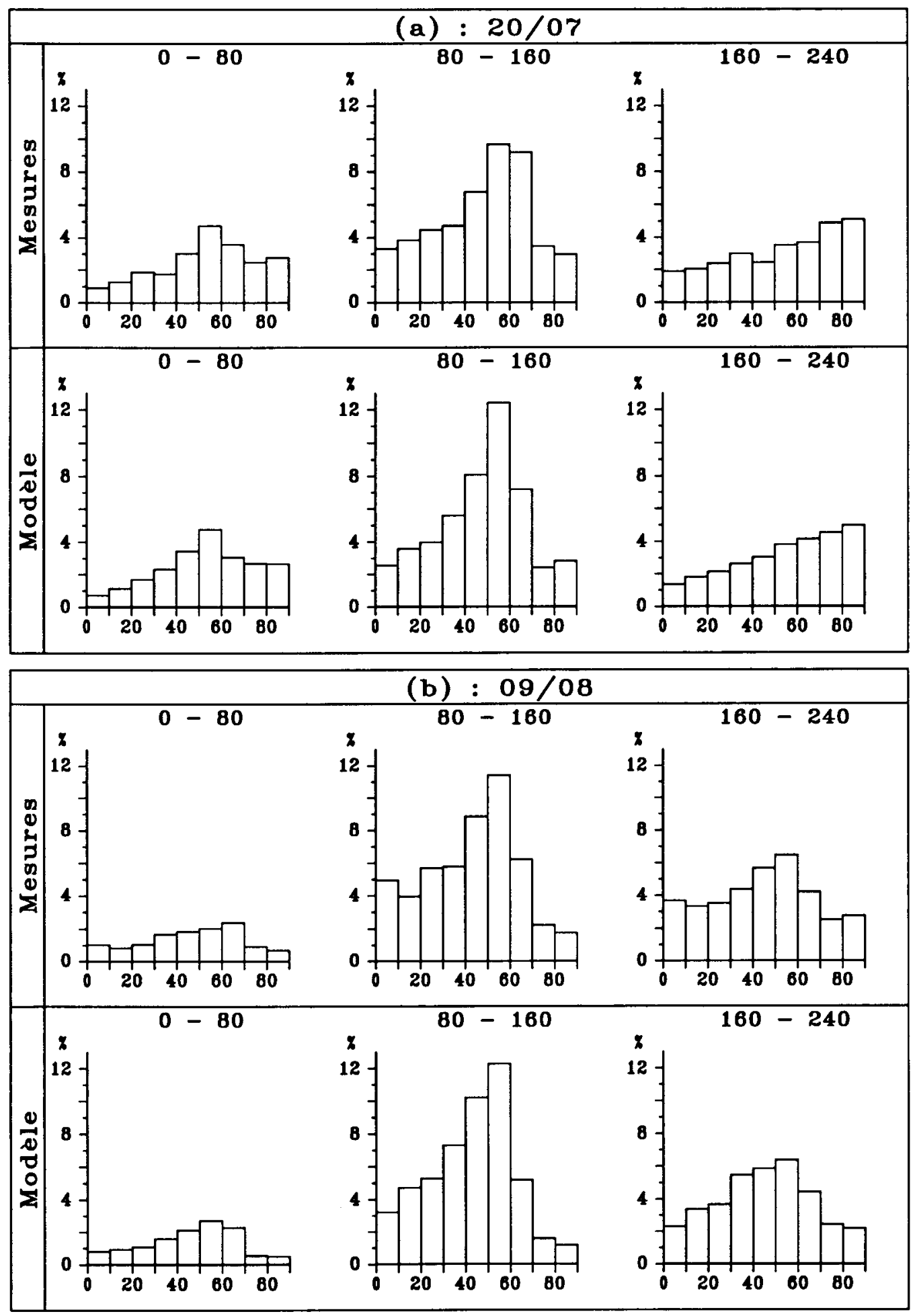

Fig 13. Fonctions de distributions des angles foliaires. Comparaison des fonctions de distribution des angles foliaires (LIDF) obtenues en appliquant la méthode des silhouettes aux mesures brutes et au modèle de nervure centrale : proportion de la surface foliaire ayant un angle d'inclinaison donné (par classes de $10^{\circ}$ ). La méthode des silhouettes est appliquée à des couches horizontales de $80 \mathrm{~cm}$ d'épaisseur; les résultats sont exprimés en pourcentage de la surface foliaire totale. (a) : données du 20 juillet; (b) : données du 09 août.

(dominance de surfaces foliaires à angle d'inclinaison élevé). Au 20 juillet, la strate supérieure de la végétation $(1,6-2,4 \mathrm{~m})$ se différencie du reste du couvert par un comportement nettement érectophile; cette caractéristique disparaît le 09 août.

Le modèle a tendance à surestimer légèrement le mode de la distribution des angles fo- 
liaires en régularisant la forme de cette distribution. Toutefois, les différences observées entre mesures et modèle sont de l'ordre de grandeur des incertitudes expérimentales et le modèle restitue la fonction de distribution des angles foliaires de façon tout à fait satisfaisante.

\section{CONCLUSION}

La modélisation présentée dans cette étude et testée sur un double jeu de données expérimentales permet de décrire avec une grande précision la structure géométrique de pieds de maïs. Les différents sous-modèles utilisés sont des formes paramétriques simples, des coniques, et ne font intervenir qu'un nombre limité de paramètres.

La feuille développée est décrite par 3 paramètres : sa longueur, un paramètre reliant sa plus grande largeur à sa longueur et un paramètre de forme, relié à son degré d'engainement. La longueur de la feuille est elle-même reliée à la hauteur de son point d'insertion.

La nervure centrale est décomposée en une partie ascendante parabolique et une partie descendante ellipsoïdale, éventuellement absente. Elle est représentée par la longueur de la feuille, la position de son point d'insertion et par 6 paramètres de forme : angle d'insertion et courbure de la partie parabolique, position du point de raccordement et angle de cassure en ce point, allongement et courbure angulaire de la partie ellipsoïdale.

Malgré ce faible nombre de paramètres, l'aptitude du modèle à représenter des formes très variées de feuilles est excellente. II permet en particulier de décrire avec une bonne précision la fonction de distribution des angles foliaires, à partir d'un nombre limité de points de mesure de la forme de la nervure centrale : 7 points par feuille sont théoriquement suffisants.

Cette modélisation doit ainsi pouvoir être utilisée avec profit en entrée de modèles de transferts radiatifs au sein des couverts végétaux, qui nécessitent une connaissance détaillée de l'architecture de la végétation, notamment pour les version bi- ou tridimensionnelles de ceux-ci.

Bien que cela n'ait été fait que pour 2 dates proches dans cette étude, il est possible de caractériser l'évolution temporelle de la structure géométrique du feuillage de pieds de maîs, en étudiant l'évolution des différents paramètres intervenant dans cette modélisation.

Enfin, la régularité des fonctions de distribution de ces paramètres doit permettre de générer, par tirage aléatoire dans ces lois, des maquettes informatiques tridimensionnelles de couverts de maïs.

\section{RÉFÉRENCES}

Aries F, Monestiez P, de Reffye P, Prévot L (1987) Modélisation de la géométrie d'un couvert végétal : cas du Maïs. In : $3^{e}$ Colloque MARI87 (Machines, Réseaux Intelligents), Paris, 197-202

Baret $F$ (1986) Contribution au suivi radiométrique de cultures de céréales. Thèse de Doctorat, université Paris XI Orsay, $182 p$

Brown PS, Pandolfo JP (1969) An equivalent-obstacle model for the computation of radiation flux in obstructed layers. Agric Meteorol 6, 407-421

Bonhomme R, Varlet-Grancher C (1978) Estimation of gramineous crop geometry by plant profiles including leaf with variations. Photosynthetica 12, 193196

Chiapale JP (1975) A numerical model for estimating the modification of heat budget introduced by hedges. In: Heat and mass transfer in the biosphere. Part I. Transfer processes in the plant environment, Dubrovnic (YUG), 457-466

Daughtry CST, Hollinger SE (1984) Cost of measuring leaf area index of corn. Agron J76, 836-841

Goel NS, Grier T (1988) A model for radiative transfer in heterogeneous three-dimensional canopies. In: Signatures spectrales d'objets en télédétection, $4^{\theta}$ Colloque International, European Space Agency. INRA-CNES-CNRS-ESA, Aussois (Poster) 133-136

Kimes DS (1984) Modeling the directional reflectance from complete homogeneous vegetation canopies with various leaf-orientation distributions. J Opt Soc Am 1, 725-737

Kimes DS, Kirchner JA (1982) Radiative transfer model for heterogeneous 3-D scenes. Appl Optics $21,4119-4129$

Marquardt DW (1963) An algorithm for least square estimation of non linear parameters. SIAM $J 11$, 431-441

Prévot L (1985) Modélisation des échanges radiatifs au sein des couverts végétaux, application à la télédétection, validation sur un couvert de maïs. Thèse de Doctorat, univ Paris VI, 178 p

Reffye (de) P, Edelin C, Françon J, Jaeger M, Puech C (1988) Plant models faithful to botanical structure and development. Computer Graphics 22, 151-158 
Riou C, Valancogne C, Pieri $P$ (1989) Un modèle simple d'interception du rayonnement solaire par la vigne - vérification expérimentale. Agronomie 9, 441-450

Sinoquet H (1988) Modélisation des échanges radiatifs de courte longueur d'onde dans certains couverts végétaux hétérogènes. Thèse de DocteurIngénieur, INA Paris-Grignon

Sinoquet $H$ (1989) Modélisation de l'interception des rayonnements solaires dans une culture en rangs. I. Aspects thèoriques. Agronomie 9, 125-135
Sinoquet H, Bonhomme R (1989) Modélisation de l'interception des rayonnements solaires dans une culture en rangs. II. Structure géométrique du couvert et validation du modèle. Agronomie 9, 619-628

Suits GH (1972) The calculation of the directional reflectance of vegetative canopy. Remote Sensing Environ 2, 117-125

Verhoef W (1984) Light scattering by leaf layers with application to canopy reflectance modeling: the SAIL model. Remote Sensing Environ 16, 125-141 